\title{
Autonomously Learning One-To-Many Social Interaction Logic from Human-Human Interaction Data
}

\author{
Amal Nanavati* \\ amaln@cs.uw.edu \\ University of Washington \\ Seattle, USA
}

\author{
Malcolm Doering ${ }^{\dagger}$ \\ doering@i.kyoto-u.ac.jp \\ Kyoto University \\ Kyoto, Japan
}

\author{
Dražen Brščić ${ }^{\dagger}$ \\ drazen@i.kyoto-u.ac.jp \\ Kyoto University \\ Kyoto, Japan
}

\author{
Takayuki Kanda ${ }^{\dagger}$ \\ kanda@i.kyoto-u.ac.jp \\ Kyoto University \\ Kyoto, Japan
}

\begin{abstract}
We envision a future where service robots autonomously learn how to interact with humans directly from human-human interaction data, without any manual intervention. In this paper, we present a data-driven pipeline that: (1) takes in low-level data of a human shopkeeper interacting with multiple customers (28 hours of collected data); (2) autonomously extracts high-level actions from that data; and (3) learns - without manual intervention - how a robotic shopkeeper should respond to customers' actions online. Our proposed system for learning the interaction logic uses neural networks to first learn which customer actions are important to respond to and then learn how the shopkeeper should respond to those important customer actions. We present a novel technique for learning which customer actions are important by first learning the hidden causal relationship between customer and shopkeeper actions. In an offline evaluation, we show that our proposed technique significantly outperforms state-of-the-art baselines, in both which customer actions are important and how to respond to them.
\end{abstract}

\section{CCS CONCEPTS}

- Computing methodologies $\rightarrow$ Learning from demonstrations; $\bullet$ Human-centered computing $\rightarrow$ HCI theory, concepts and models.

ACM Reference Format:

Amal Nanavati, Malcolm Doering, Dražen Brščić, and Takayuki Kanda. 2020. Autonomously Learning One-To-Many Social Interaction Logic from Human-Human Interaction Data. In Proceedings of the 2020 ACM/IEEE International Conference on Human-Robot Interaction (HRI '20), March 2326, 2020, Cambridge, United Kingdom. ACM, New York, NY, USA, 9 pages. https://doi.org/10.1145/3319502.3374798

\section{INTRODUCTION}

As service robots get deployed in public spaces, they are increasingly coming in contact with human groups. How such robots should socially interact with multiple humans is an open question.

\footnotetext{
${ }^{*}$ Formerly affiliated with Kyoto University and ATR.

${ }^{\dagger}$ Also with Advanced Telecommunications Research Institute International (ATR).

Permission to make digital or hard copies of all or part of this work for personal or classroom use is granted without fee provided that copies are not made or distributed for profit or commercial advantage and that copies bear this notice and the full citation on the first page. Copyrights for components of this work owned by others than ACM must be honored. Abstracting with credit is permitted. To copy otherwise, or republish, to post on servers or to redistribute to lists, requires prior specific permission and/or a fee. Request permissions from permissions@acm.org.

HRI '20, March 23-26, 2020, Cambridge, United Kingdom

(C) 2020 Association for Computing Machinery.

ACM ISBN 978-1-4503-6746-2/20/03 . .\$15.00

https://doi.org/10.1145/3319502.3374798
}

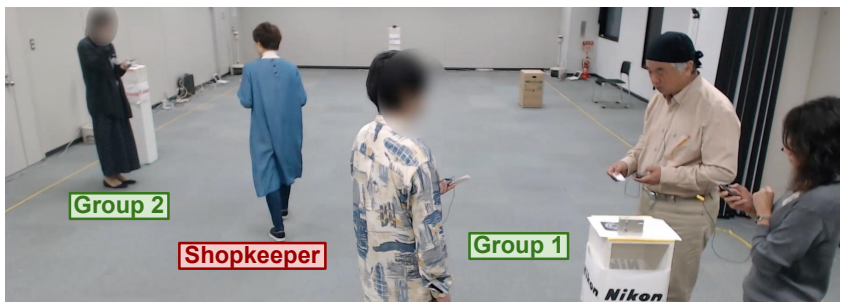

Figure 1: A shopkeeper interacting with many customers.

We are also moving towards an Internet of Things world, where internet-connected sensors passively collect data about how humans interact with one another. This creates an opportunity for service robots to leverage that passively collected human-human interaction data to learn how to interact with humans. In this work, we are particularly interested in the interactions between a service professional and multiple people, who may form multiple spatially separated groups, such as customers in a store.

While much research has been devoted to learning interaction logic from human-human data for one-to-one scenarios [7, 20, 21], one-to-many scenarios introduce novel challenges. Consider Fig. 1. The shopkeeper was talking to Group 1. She had to respond to multiple customers' queries and distinguish between utterances directed at other group members (e.g., "What do you think?") and those directed at her (e.g., "How much is this?"). After answering Group 1's questions, the shopkeeper goes to help Group 2, who had asked for her help previously. To do this, the shopkeeper had to keep track of customer actions, prioritize customer needs, and determine when to approach/leave customers. Finally, as she answers Group 2's questions, customers in Group 1 continue talking amongst themselves. Therefore, the shopkeeper must handle asynchroneities in actions across customer groups. These nuances do not exist in one-to-one scenarios, and make it difficult to manually program a robot to handle one-to-many interactions. Therefore, we propose autonomously learning the interaction logic for one-tomany interactions from human-human data.

In this paper, we present a data-driven pipeline that autonomously learns - without explicit manual labeling - how a shopkeeper should respond to multiple customers' actions online. Specifically, our system uses neural networks to first learn which customer actions are important to respond to (Attention Network) and then learn how the shopkeeper should respond to those important customer actions (Interaction Network). We train the Attention Network by first learning which customer actions likely caused shopkeeper reactions (Causal Inference Network). We show that: 
(1) First learning which customer actions are important and then learning how to respond to them significantly improves the social appropriateness of predicted shopkeeper actions;

(2) Training the Attention Network by first learning which customer actions likely caused shopkeeper reactions significantly improves the Attention Network's accuracy.

Our dataset and neural network code can be found here ${ }^{1}$.

\section{RELATED WORK}

Data-driven techniques are becoming increasingly common in human-robot interaction (HRI) research. Past works have used data to develop specific components of a robotic system: gaze [24], nonverbal behaviors [1], turn-taking [27], motion prediction [23], engagement prediction [2], and information transfer perception [32]. In some works, researchers analyze the data and hand-craft a system that displays the desired capabilities [23, 24, 27, 32]. In others, a human manually labels the data and uses it to train a system that displays the desired capabilities [1]. In yet others, a system autonomously perceives labels and uses them to learn the desired capabilities [2]. Like the latter, our learning pipeline is fully automated and requires no explicit labeling. Finally, some data-driven works focus on learning most of a robot's interaction capabilities directly from data, but they chiefly collect data from virtual worlds that lack the complexities of human-human interactions [5, 25].

The HRI community has also been focusing on scenarios where a robot interacts with multiple humans. Robots explicitly developed to handle multi-party interactions include a bartender robot [15], a caretaker robot [26], a guide robot [28], and an embodied conversational agent [4]. These works and others often focus on a robot's ability to handle a specific aspect of multi-party interactions: receiving and responding to multiple requests $[15,26]$, group detection [28, 29], speech recognition [10, 11], gesture generation [18], body orientation generation [30], gaze generation [4], etc. Relevant studies in multi-party turn-taking $[3,14]$ use hand-crafted features (e.g., whether someone is speaking, head pose, prosody) to determine when the robot should take a turn, but do not incorporate the contents of speech. The closest multi-party work to ours, [15], uses human-human and human-robot data that was manually labeled to learn low-level submodules for how a bartender robot should interact with multiple customers (e.g., classifying user engagement, or saying pre-defined utterances). Our work, on the other hand, requires no explicit labeling and learns high-level interaction logic.

Past works from our lab have focused on learning most of a robot's interaction capabilities directly from human-human interactions, without explicit labeling [7, 8, 20,21]. One such research direction focused on one-to-one shopkeeper-customer interactions. Those works demonstrated success transferring interaction capabilities learnt from human-human data into a human-robot setting [20], extended that robotic shopkeeper's capabilities by making it proactive [21], and enabled the robotic shopkeeper to use curiosity to modify its online behavior [8]. The data-driven approach has also been applied to a travel agent scenario [7]. In this paper, we directly build upon this research direction by extending it from a one-to-one to a one-to-many shopkeeper-customer scenario.

\footnotetext{
${ }^{1}$ http://www.robot.soc.i.kyoto-u.ac.jp/en/research/dataset-one-to-many-humanhuman-interaction/
}

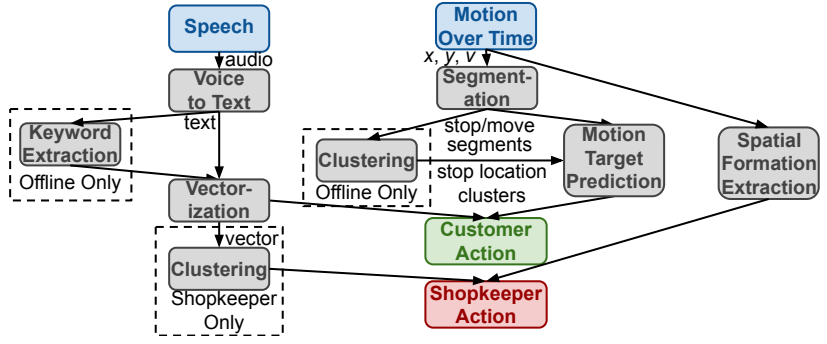

Figure 2: The data processing pipeline converts audio and motion data into discrete customer and shopkeeper actions.

\section{DATA COLLECTION AND PROCESSING}

\subsection{Data Collection}

We set up a medium-sized $(8 \times 20 \mathrm{~m})$ lab space to look like a camera shop, invited participants to role-play shopkeeper-customer interactions, and recorded their audio and movements. Each data collection session had one shopkeeper - one of two recurring participants with service experience - and 2-5 customers, who each role-played multiple scenarios. We used laser range-finders and a particle filter to track participants' motion over time [9]. We also used one smartphone app and headset per person to record audio, where participants specified the start/end of their utterance on the app [20]. During data collection, a researcher specified which tracked participants were the shopkeeper/customers. To make the problem feasible to learn within time and resource constraints, we specified the camera attributes customers should mainly talk about (price, weight, color, resolution, and camera-specific features). Per scenario, we assigned customer groupings and roles - portrait photographer, nature photographer, novice photographer, curious customer, window shopper, or bored customer. These roles were merely meant to elicit various behaviors, and were not given to the learning system. In total, we had 51 participants (35 F, $16 \mathrm{M})$ and recorded 266 scenarios (28.7 hours of data), divided evenly between the two shopkeepers and roughly evenly between the number of customers (2-5) in the scenario. Data collection occurred in Japanese, and the procedure was approved by an organizational ethics review process. Neither the shopkeeper nor customers were trained or scripted in how to interact with one another. Fig. 1 shows a sample interaction during data collection.

\subsection{Data Processing}

Our dataset ${ }^{1}$ consists of a set of scenarios, each with $x, y$ position and $v$ velocity per participant over time, as well as recorded audio (a separate audio file per utterance). The goal of data processing is to autonomously convert this low-level data into a sequence of discrete, high-level shopkeeper and customer actions to serve as inputs and outputs to the learning system. Fig. 2 shows the data processing pipeline. We highlight main points and differences from past works here, and encourage readers to read [21] for more details.

3.2.1 Speech Data Processing. We began by converting each utterance's audio to text, using Google's Speech API. We then vectorized 


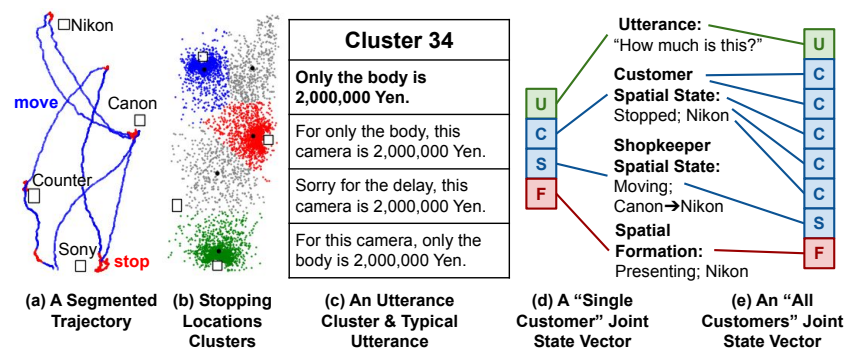

Figure 3: Sample outputs from data processing.

the text by: (1) lemmatizing the words using $\mathrm{MeCab}^{2}$; (2) extracting n-grams; (3) generating a term-frequency inverse-documentfrequency (TF-IDF) matrix; (4) running Latent Semantic Analysis (LSA) [6]; and (5) reducing the dimensionality to a $50 \%$ share of accumulated singular values [31]. We ran the same procedure on extracted keywords from each utterance (using Google Cloud's Natural Language $\mathrm{API}^{3}$ ) and appended the two vectors. We processed shopkeeper and customer speech data separately, resulting in a shopkeeper vector of size 639 and a customer vector of size 1348.

3.2.2 Spatial Data Processing. We learnt a natural threshold between moving and stopped velocities by running Jenks Natural Breaks Optimization [13] on the speed-per-timestep data. We used that threshold to segment each participants' trajectories into "moving" and "stopped" segments (Fig. 3a). For stopped segments, we clustered them using k-means [22] (Fig. 3b). We determined the best $k$ as the one with the highest second derivative. Moving forward, all references to locations indicate a stopping location cluster.

3.2.3 Customer Action Detection. Since the ultimate goal of the system is to take in customer actions that are detected in-real time and output shopkeeper responses, customer action detection must be done online. Customer actions consist of speech and motion components. For the speech components, audio files can be immediately transcribed and vectorized once the participant specifies their utterance is over (through the speech capture smartphone application). For the motion components, we predict the target of motion from the change in customer position over the last 3 seconds. Our processed dataset consists of 17, 524 customer actions (e.g., MOVE: Door $\rightarrow$ Canon; SPEECH: "This camera looks nice," etc.).

3.2.4 Shopkeeper Action Detection. Shopkeeper actions consist of a speech cluster and spatial formation. They can be extracted from the training data offline, since during execution a robotic shopkeeper always knows what action they are doing. We cluster shopkeeper utterances using dynamic hierarchical clustering [19] and extract a typical utterance (the mediod) per cluster (Fig. 3c). We then extract spatial formations based on the relative position of the shopkeeper and customers. The spatial formation is "present" if the shopkeeper and at least one customer are at the same camera, "part of group" if they are near each other away from a camera, and "waiting" otherwise. Each spatial formation is associated with the location that formation takes place in. Our processed dataset consists of 7, 600 shopkeeper actions.

\footnotetext{
${ }^{2}$ https://taku910.github.io/mecab/

${ }^{3}$ https://cloud.google.com/natural-language/
}

3.2.5 Vectorization. Inputs: We represented actions with a joint state vector containing the acting agent's utterance, customer(s) spatial state(s), the shopkeeper's spatial state, and the shopkeeper's spatial formation. Spatial states consisted of the current location (when stopped), or motion origin and target (when moving). We had two input representations, used at different steps of training. "Single customer" (Fig. 3d) contained only one customer's spatial state, while "all customers" (Fig. 3e) contained every customer's spatial state (with extra bits for which agent acted). Each input incorporated the $T:=3$ past actions, resulting in "single customer" inputs of size 3461 and "all customers" inputs of size 3692.

Outputs: We represented shopkeeper output actions using action IDs, which refer to one speech cluster and target spatial formation. Our output consisted of three one-hot vectors: whether the action contained speech (size 2); the action ID if it did not contain speech (size 12); and the action ID if it did contain speech (size 812).

\section{PROPOSED SYSTEM}

\subsection{Problem}

The goal of the proposed system is to predict shopkeeper reactions from customer actions and interaction context. Therefore, a naive approach involves learning how the shopkeeper responded to each customer action. This approach was used in the state-of-the-art system for one-to-one interactions [21]. However, such a global action-reaction construction fails to adequately model one-to-many interactions. Consider the sample interaction in Fig. 4. Fig. 4b shows the global action-reaction sequence, which assumes every customer action has a shopkeeper reaction. In this model, Customer 5's (C5) action at Time 7 is modeled as having no shopkeeper response, although the shopkeeper did respond at Time 9. Conversely, the shopkeeper action at Time 9 is modeled as a response to C4's action at Time 8 , although that action is unrelated. These problems arise due to the asynchroneity of actions across groups. Another problem with this construction is the resulting data imbalance. Because there are more customers than shopkeepers, there are many more shopkeeper "no actions," making it difficult for the system to learn meaningful associations for actual shopkeeper actions.

\subsection{Proposed Solution}

In contrast, we propose modeling the interaction as multiple separate action-reaction sequences, one per customer (Fig. 4c). Intuitively, when a shopkeeper acts, they are responding to the most recent action of at least one customer. Therefore, by separating the actions that could have triggered a shopkeeper reaction by customer, we are guaranteed that at least one action-reaction sequence accurately models the interaction at that time.

Further, this construction correctly models "no actions" (which incidentally addresses the "no action" data imbalance). Unlike other actions, "no actions" are not perceived, and must be assumed by our action-reaction model. Our proposed model accurately describes "no actions" as contingent on the customer who is important at that time. Consider Time 11 in Fig. 4c. If C3 is important, then we should assume the shopkeeper intentionally responded to C3 with "no action," since the next perceived action in that sequence is another $\mathrm{C} 3$ action. However, if Customer 2 is important, we should assume the shopkeeper's action at Time 14 was in response to C2's action 


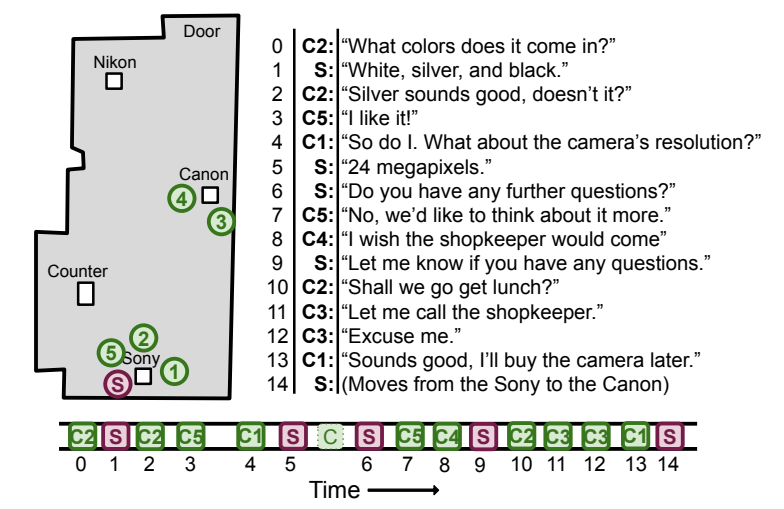

(a) Sample Interaction Sequence

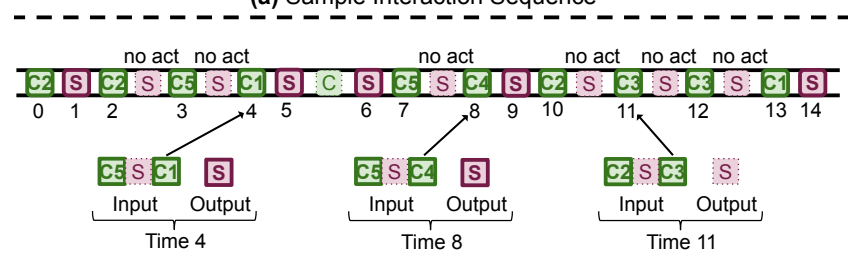

(b) Global Action-Reaction Sequence

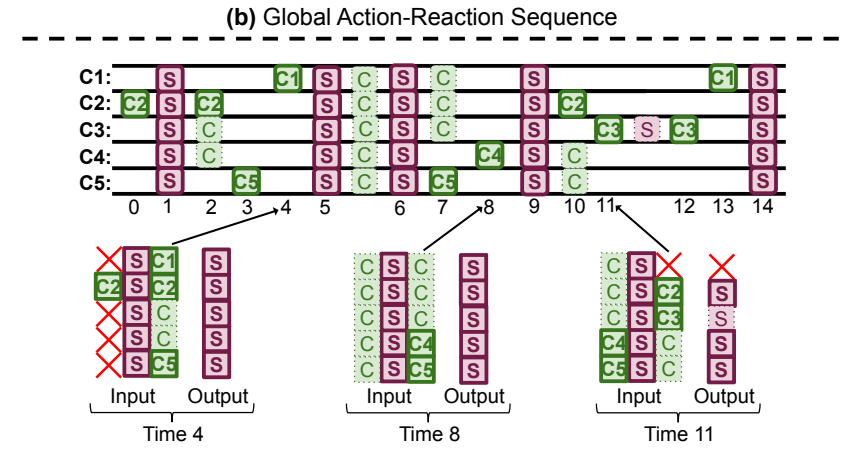

(c) Per-Customer Action-Reaction Sequences

Figure 4: A global (b) and per-customer (c) action-reaction sequence for the same example interaction (a).

at Time 10. In this way, the per-customer action-reaction sequence accurately models the interaction at every timestep, conditioned on which customer is important for the shopkeeper to respond to.

\subsection{System Overview}

Since we model the interaction as per-customer action-reaction sequences, our learning system must learn both which customer actions are important and how the shopkeeper should respond to them. Therefore, our proposed system consists of two components:

- At every timestep, the Attention Network (Fig. 5a) takes in the recent actions of all customers and outputs the index of the customer who is most important to respond to at that time. If that customer is the one who acted, that action is passed to the Interaction Network. If not, that action is filtered out and the shopkeeper responds with "no action."

- When the customer who acted is deemed to be important, the Interaction Network (Fig. 5b) takes in only the important customers' recent actions and outputs a shopkeeper reaction.

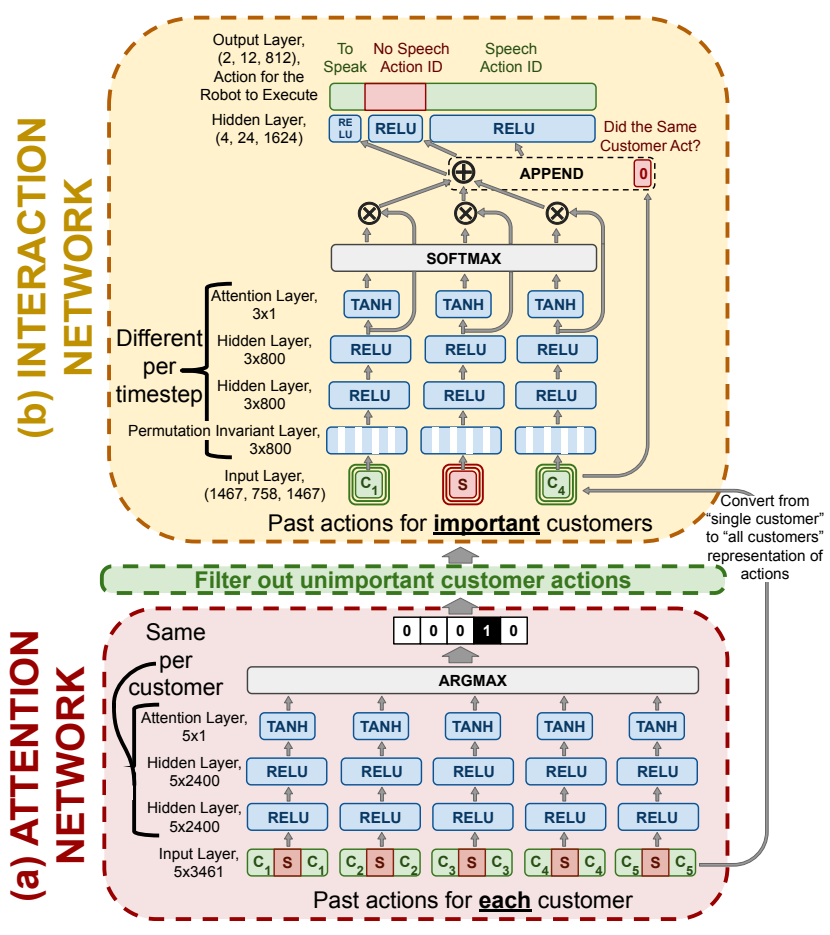

Figure 5: Our proposed system consists of an Attention Network (a) and Interaction Network (b).

\subsection{Attention Network}

After every customer action, the Attention Network (Fig. 5a) takes in the $T:=3$ most recent actions per customer, using the percustomer action-reaction sequences model (Figure 4c). It passes each customer's sequence through two rectified linear unit (RELU) hidden layers and one hyperbolic tangent (TANH) layer to get an importance score for that customer. It then takes the argmax of that score to determine the most important customer. If the most important customer is the one who acted at that timestep, their action is passed on to the Interaction Network. If not, it is ignored, resulting in the robotic shopkeeper not responding to that customer action (e.g., when a customer in another group says something to their friend). To ensure that the network is permutation-invariant across customer ID, the weights of the layers are the same for all customers. Importantly, since the number of customers is variable, the Attention Network masks out any customers not present. Since the Attention Network takes in all customers' actions, it only needs to include one customer's spatial state per action; hence, it uses the "single customer" representation of joint state vectors (Sec. 3.2.5).

\subsection{Interaction Network}

The Interaction Network (Fig. 5b) takes in the recent $T:=3$ important actions (i.e., the two most recent important customer actions, and the shopkeeper action in-between them) and outputs a shopkeeper reaction. It models shopkeeper and customer actions like the global action-reaction sequence does (Fig. 4b), although it only uses important customer actions. Since the Interaction Network maximally takes in two customers' actions, it can benefit from 
knowing other customers' spatial states; hence, it uses the "all customers" representation of joint state vectors (Sec. 3.2.5). As with the state-of-the-art system [21], it passes each action through two RELU hidden layers, followed by an attention-over-time mechanism using a TANH layer. However, unlike the state-of-the-art system, we first pass each action through a permutation-invariant layer [33], which separates each customer's spatial state, puts each through the same hidden layer, and averages them. This results in permutation-invariance with regards to customer ID within each timestamp. After the attention over-time mechanism, we append a bit to the data that indicates whether the same or different customers acted across the two customer actions; this makes the network permutation-invariant across timestamps. Finally, after the attention over time mechanism, the data goes through two more RELU layers, split per component of the output (Sec. 3.2.5): whether the shopkeeper should speak, the no speech action ID (the system's output if the shopkeeper should not speak), and the speech action ID (the system's output if the shopkeeper should speak). Finally, we take the argmax of each component, to get the one predicted action ID.

\section{TRAINING THE PROPOSED SYSTEM}

\subsection{Problem}

The proposed system cannot be trained with standard gradientbased methods, due to the non-differentiable argmax and filter between the Attention and Interaction Networks. Each network can be trained separately with gradient-based approaches, but this requires ground-truth labels for the Attention Network, which we do not have. One approach involves manual labeling who the shopkeeper should pay attention to. However, that does not align with our goal of developing a fully autonomous data-driven pipeline. Another approach involves modifying the system architecture to jointly train the Attention and Interaction Networks. However, as we demonstrate in Sec. 6.2.2, this produces poor results.

\subsection{Proposed Solution}

Although we do not have ground-truth attention labels, we do have ground-truth action labels. We know the shopkeeper's action, and assume she acted in response to a customer action. Thus, the shopkeeper's action should be most highly correlated with the customer action that caused it, according to some hidden correlation function.

Therefore, we propose a solution that learns the attention labels using causal inference. Specifically, for every shopkeeper action, we propose learning which customer most likely caused the shopkeeper action, and assume that is the customer to pay attention to right before the shopkeeper acted (Fig. 6a). We then extrapolate those attention labels backwards till the previous shopkeeper action (Fig. 6b). Our rationale for this is that had another customer been important for the shopkeeper to respond to, the shopkeeper would have responded to that customer instead.

\subsection{Causal Inference Network}

We learn the aforementioned causal relation between customer and shopkeeper actions using the Causal Inference Network (Fig. 7). It takes in the per-customer action-reaction sequences at timesteps before a shopkeeper acts. It begins by passing these inputs through
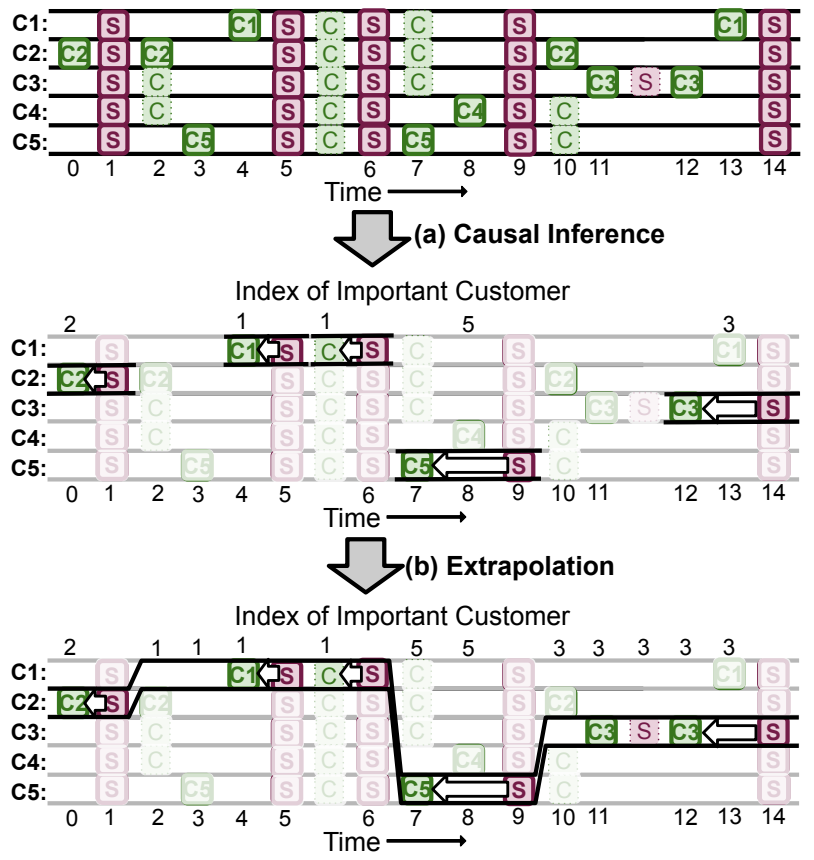

Figure 6: Our proposed solution for learning attention labels

layers with the same architecture as the Attention Network. However, instead of taking the argmax of importance scores, it draws a sample from the differentiable Gumbel-Softmax distribution [12]. This results in continuous weights that tend to approximate the argmax of the importance scores. Then, instead of filtering out unimportant customer actions, the network uses those attention weights to take a weighted average of the inputs (after they go through a RELU hidden layer that accounts for the data's temporal structure). This results in a hidden representation of a customer, shopkeeper, and customer action, mostly consisting of the important customer's actions. The network then passes these values through layers with the same architecture as the Interaction Network (except for the "did the same customer act" bit and the "all customers" representation - these are unnecessary since the weighted average includes part of every customer's action). Therefore, the network outputs a shopkeeper action. However, the importance scores computed within the network - the input to the Gumbel-Softmax distribution - implicitly specify which customer action most predicted the shopkeeper reaction - in other words, which customer action likely caused the shopkeeper reaction. Note that although the Causal Inference Network seems to merely be a differentiable version of the proposed system, the main difference is that it is only trained on timesteps before a shopkeeper action.

\subsection{Training Methodology}

We train the networks sequentially. We first train the Causal Inference Network and extrapolate the attention labels (the argmax of the learned importance scores) to all timesteps (Fig. 6). We then use those attention labels to train the Attention Network. Finally, we filter out unimportant customer actions based on the Attention 


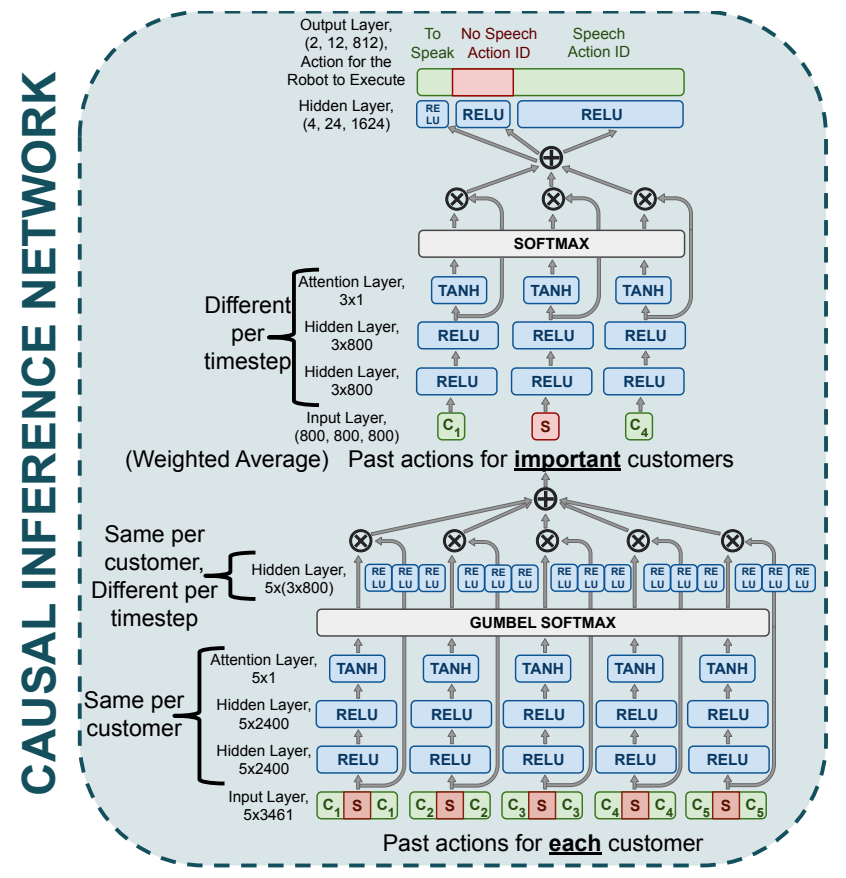

Figure 7: The Causal Inference Network, a helper network that generates labels to train the Attention Network.

Network's output, re-establish an action-reaction sequence (that only contains important customer actions), and train the Interaction Network. All layers except the last two have hidden layer sizes of 800 per customer/shopkeeper action, as in [21] - we tried 1200, but found that the resulting networks overfit more. We train all networks with the cross-entropy loss. For the Interaction Network and Causal Inference Networks, the loss is the cross-entropy loss of the "to speak" component, added with either the cross-entropy loss of the "speech action ID" component or the cross entropy loss of the "no speech action ID" component, depending on whether the ground truth label's action contains speech. All networks were trained with the Adam optimizer [16] on randomized minibatches of size 128. The interaction networks (both the Interaction Network and the interaction component of the Causal Inference Network) had a learning rate of $10^{-4}$, while the attention networks (both the Attention Network and the attention component of the Causal Inference Network) had one of $2 \times 10^{-5}$. All networks were trained until minimum validation loss, which happened within 150 epochs. For the Causal Inference Network, we annealed the Gumbel-Softmax temperature from 2.0 (closer to softmax) to 0.25 (closer to argmax) at a rate of -0.75 per 10 epochs. Layers that did not directly take in actions used a dropout rate of 0.5 .

\section{EVALUATION}

We conducted two offline evaluations, corresponding to the two main predictions of our proposed system: the system output (predicted shopkeeper response) in Sec. 6.1; and the attention output (which customer action is important) in Sec. 6.2.

\subsection{Output Shopkeeper Action Correctness}

6.1.1 Procedure. To investigate the effect of the Attention Network on system outputs, we compared our proposed system with the same system without the Attention Network. Proposed is the complete system (Secs. 4 and 5), while Without Attention is only the Interaction Network. Since it doesn't use the Attention Network, Without Attention models the interaction with a global action-reaction sequence (Fig. $4 \mathrm{~b}$ ).

Both systems were trained using hold-one-out cross-validation on the collected scenarios. Specifically, of the collected 266 scenarios (where each scenario contains many actions), we trained each system on 257 scenarios until they reached minimum loss on a validation set of 8 scenarios, and held 1 scenario out for testing. We repeated this procedure 14 times, so our test sets accounted for $5 \%$ of the collected scenarios. The 14 test sets and every validation set were balanced across: (1) number of customers in the scenario; and (2) which of the two participants was role-playing as the shopkeeper. The same test sets were used across both systems. Overall, the test sets across all 14 folds of cross-validation consisted of 881 customer actions, while each training set consisted of 16, $900-17,000$ (depending on the held-out scenario).

For each customer action, we needed to evaluate the correctness of two predicted shopkeeper responses (one per system). One way to evaluate correctness uses prediction accuracy. However, for a social interaction, actions with different action IDs could still be socially equivalent. For example, "It has 9 preset modes" and "The Sony has 9 preset modes" are assigned different action IDs, but are both appropriate responses to the customer utterance "Does this camera have preset modes?" Therefore, the metric of action ID accuracy would under-evaluate correctnesses. To account for this, we instead evaluate the systems' "social appropriateness," as in [21]. A shopkeeper action is socially appropriate if and only if it is both factually correct and appropriate given the social context.

We used a human coder to measure the social appropriateness of both systems' predicted shopkeeper actions. For every customer action, the human coder was shown all actions leading to that action, and the Proposed and Without Attention systems' predicted responses (randomly ordered). They were then asked to separately label whether each response was socially appropriate, and were instructed to label factually incorrect answers as inappropriate. To check coder reliability, we had a second coder independently rate $10 \%$ of the data. The two coders had a Cohen's $\kappa$ value of 0.87 , indicating that it was reliable to proceed with one coder.

6.1.2 Results. Overall, the Proposed system output more socially appropriate actions than the Without Attention system. We analyzed the statistical significance of this result with McNemar's test for paired binary data on the human coder's social appropriateness ratings, removing timesteps where the coder was "unsure" of how the shopkeeper should respond. Table 1 shows the results of this analysis, which revealed a significant difference $\left(\chi^{2}(1, N=850)=51.05, p<0.001\right)$ between the two systems. The Proposed system was correct $77.0 \%$ of the time, compared to the Without Attention system's 65.2\%.

A manual analysis of system outputs revealed two significant weaknesses to the Without Attention system, which the Proposed system addressed. First, the Without Attention system 


\begin{tabular}{|c|c|c|c|c|c|c|}
\hline$\sqrt{\text { Nikon }} \sqrt{\text { Door }}$ & Customer ID & Customer Action & Proposed Response & Without Attention Response & $\begin{array}{l}\text { Proposed } \\
\text { Attention }\end{array}$ & $\begin{array}{l}\text { Without Causal } \\
\text { Inference Attention }\end{array}$ \\
\hline & 2 & $\begin{array}{l}\text { "I intend to take pictures on my upcoming } \\
\text { vacation, which is why I came today." }\end{array}$ & un & ""” & 2 & 2 \\
\hline (2) & 3 & "May I ask you a question?" & (Move to Nikon) & (Move to Nikon) & 2 & 2 \\
\hline & 3 & $\begin{array}{l}\text { "I like taking pictures while in nature; which } \\
\text { camera would be good for that?" }\end{array}$ & $\begin{array}{l}\text { "This is the Nikon camera, } \\
\text { which at } 120 \mathrm{~g} \text { is very light" }\end{array}$ & " & 3 & 2 \\
\hline & 2 & $\begin{array}{l}\text { "I'll be taking pictures of my friends so want } \\
\text { a camera that takes glamorous portraits." }\end{array}$ & "” & $\begin{array}{l}\text { "This has } 12 \text { glamor retouch effects, } \\
\text { like soft skin, small face, and big eyes" }\end{array}$ & 3 & 1 \\
\hline Nikon Door & & & & & & \\
\hline (1) & 1 & "What is the price of this camera?" & "The price is 6,800 Yen" & "” & 1 & 4 \\
\hline $\int \quad$ Canon & 4 & $\begin{array}{l}\text { "This camera doesn't seem like it has silent } \\
\text { shooting" }\end{array}$ & "” & "The price is 6,800 " & 1 & 1 \\
\hline${ }^{\text {Counter }}$ & 5 & $\begin{array}{l}\text { "Yeah, it's important for it to have silent } \\
\text { shooting" }\end{array}$ & "” & "” & 2 & 2 \\
\hline 7 (4) & 2 & "That's really affordable!" & "Yes, that is right" & "At 120 grams, it is very lightweight" & 2 & 5 \\
\hline
\end{tabular}

Figure 8: Sample evaluation results (translated to English), and whether the human coder labeled them as correct or wrong.

\begin{tabular}{|c|c|c|c|c|}
\hline & \multicolumn{2}{|c|}{ Without Attention } & \multirow[b]{2}{*}{ Total } \\
\hline & & Correct & Wrong & \\
\hline \multirow{3}{*}{ Proposed } & Correct & 0.598 & 0.172 & 0.770 \\
\hline & Wrong & 0.054 & 0.176 & 0.230 \\
\hline & Total & 0.652 & 0.348 & 1.00 \\
\hline
\end{tabular}

Table 1: The proportion of shopkeeper actions that were labeled socially appropriate or inappropriate, per model.

was poor at determining which customer to respond to. For example, in Fig. 8 Top, although Customer 2 is far away from the shopkeeper, the Without Attention system picks up on the keyword "glamorous" and has the shopkeeper respond to them. Second, it overfits to asynchroneities. For example, in Fig. 8 Bottom, the Without Attention system does not respond to Customer 1's query until after another customer has said an unrelated utterance. The Proposed system avoids both these shortcomings, since the Attention Network filters out unimportant customer actions and therefore prevents the Interaction Network from being trained on irrelevant customer actions or asynchroneities.

These results show that adding the Attention Network significantly improves the proposed system. In other words, reasoning about who to pay attention to significantly improves the social appropriateness of output shopkeeper actions, by enabling the system to handle asynchroneities in one-to-many interactions.

\subsection{Attention Accuracy}

6.2.1 Procedure. To investigate the effect of our training procedure on the attention mechanism, we compared another two systems. Proposed is the complete system (Secs. 4 and 5), while Without Causal Inference consists of an attention mechanism that is not trained using the Causal Inference Network. Instead, Without Causal Inference jointly learns attention over customers and how the shopkeeper should respond to that customer. It does so by using the same architecture as the Causal Inference Network, but on all timesteps as opposed to those directly before shopkeeper

\begin{tabular}{c|c|c|c|c}
\multicolumn{2}{c}{} & \multicolumn{2}{c}{ Without Causal Inference } \\
\cline { 3 - 5 } Proposed & Correct & Wrong & Total \\
\cline { 2 - 5 } & Correct & 0.605 & 0.302 & 0.907 \\
\cline { 2 - 5 } & Wrong & 0.010 & 0.083 & 0.093 \\
\cline { 2 - 5 } & Total & 0.615 & 0.385 & 1.00
\end{tabular}

Table 2: The proportion of timesteps whose attention was labeled as correct or not, per model.

actions. For both Proposed and Without Causal Inference, we only focus only on the outputs of the attention component.

Both systems were trained with the cross-validation procedure specified in Sec. 6.1.1. To gather ground-truth attention labels for evaluating attention accuracy, we added an additional question to the aforementioned coding tool. Therefore, the human coder also labeled which customer(s) were important for the shopkeeper to pay attention to at each timestep. The fuzzy $\kappa$ [17] measure of inter-coder reliability across the two coders was 0.70 on $10 \%$ of the data, indicating that it was reliable to proceed with one coder.

6.2.2 Results. Overall, the Proposed system had higher attention accuracy than the Without Causal Inference system. We compared the systems by giving each attention output a score of 1 if the customer it deemed was important was also deemed important by the human coder, and 0 otherwise. We then performed McNemar's test for paired binary data (Table 2 ), which revealed a significant difference between the systems $\left(\chi^{2}(1, N=881)=238.31, p<0.001\right)$. In particular, the Proposed system was correct $90.7 \%$ of the time, compared to the Without Causal Inference system's 61.5\%.

A manual analysis of attention predictions revealed that the Without Causal Inference system often predicted customers who were far away from the shopkeeper (Fig. 8 shows sample predictions). This is largely due to the fact that at many timesteps, how a shopkeeper should respond to a customer depends on which customer is important. Refer back to Fig. 4c. At Time 11, if Customer 3 is the important customer, then the output label is "no action," but if Customer 2 is the important customer, then the output label is the perceived shopkeeper action. Therefore, the attention 
weights learnt by the jointly trained network (Without Causal Inference) not only select an input to the interaction component of the network, but also an output label. In other words, the attention component of the jointly trained network can modify the output label that it itself is trained on, which opens the way for the network to learn unintended and poor strategies. The Proposed system avoids this pitfall by training the Attention Network on a separate loss function from the Interaction Network.

This result shows that our training procedure (using the Causal Inference Network and then extrapolating labels) significantly improved attention accuracy, resulting in the high accuracy of $90.7 \%$.

\section{DISCUSSION}

\subsection{Alternative Approaches}

Although the results show that our proposed system works well, one could consider alternate system architectures for learning the interaction logic of one-to-many interactions. We tried many such systems before settling on the proposed one. For example, we tried a system that took in the global action-reaction sequence, first learned whether the shopkeeper should respond or not, and then learnt how the shopkeeper should respond. However, as with the Without Attention system (Sec 6.1), we found that the system overfit to asynchroneities in customer actions. We also tried multiple deterministic alternatives to the Causal Inference Network. Assuming that the closest customer caused the action yielded $76 \%$ accuracy, assuming that the customer who acted most recently caused the action yielded 78\% accuracy, while the Causal Inference Network yielded $88 \%$ accuracy. Finally, we considered using additional modalities such as eye gaze, but decided to stick with spatial and speech data because extracting it is more reliable given existing perception capabilities. However, our approach can be extended to modalities such as eye gaze, given a way to detect discrete actions in that modality (e.g., "C1 looked at the Shopkeeper").

\subsection{Generalizability}

7.2.1 To a Physical Robot. A limitation of this work is that we evaluated the proposed system offline, and not on a physical robot. This raises the question of whether our trained policy - which learns how a human shopkeeper should respond to human customers will be applicable to a robotic shopkeeper. How human customers interact with a robotic shopkeeper will likely be different from how they interact with a human shopkeeper, which could give rise to errors that compound over time. However, past works for one-to-one interactions $[20,21]$ found that such a data-driven approach effectively transferred to a human-robot scenario. Notably, [20] found that only $7 \%$ of robot errors were due to differences in human-robot and human-human interactions (e.g., customers not saying "excuse me" to the robot). This makes us hopeful that our system will generalize to a real robot. However, investigating whether the oneto-many interaction gives rise to novel transferrability challenges is an important direction for future work.

7.2.2 To the Real-World. Another limitation of this work is the in-lab nature of our data collection. The presented data-driven approach is intended for service scenarios with repeatable interaction patterns (e.g., shopkeeper-customer, tour guides, etc.), and will learn those repeatable patterns (e.g., greetings, product-specific questions, etc.). We believe those core interaction patterns will still manifest in the real-world, albeit with greater variability that will require more data in order for this approach to generalize.

Our data-driven pipeline was explicitly designed to scale to the more complex scenarios one may expect in the real-world. For example, the number $k$ of stopping locations is learnt, the size of utterance vectors is a function of the latent topics learnt by LSA, and the number of shopkeeper clusters is adaptive based on the utterance vector distribution. Further, the Attention Network consists of the same modules - that calculate importance scores - repeated per customer. Therefore, to account for more than 5 customers, one merely needs to add more modules and train the Attention Network on data with the appropriate number of customers.

Despite collecting data in-lab, our dataset was not perfect and had noise. $12 \%$ of the utterances had speech recognition errors, $2 \%$ had cross-talk (multiple people's utterances merging), and $4 \%$ had incomplete utterances. The detected $x, y$ positions had variance, and the person tracker sometimes lost participants. Our abstractions and clustering allowed the system to perform well despite noise, which is a positive sign for its generalizability to the real-world.

Finally, our pipeline does rely on forms of weak labeling. For example, the participants specified when their utterances started and stopped, and a researcher specified which tracked participant was the shopkeeper. However, these are merely stop-gap measures, and can be automated as the appropriate technologies advance (e.g., multi-party speech recognition and human role detection).

We would like to point out that our proposed system's 77\% social appropriateness is higher than past works (which handled the simpler problem of one-to-one interactions) [21]. We believe these results would be improved with more data and a less noisy speech recognition system.

\section{CONCLUSION}

In this paper, we presented a system that autonomously learns interaction logic in a one-to-many shopkeeper-customer scenario from human-human data, without explicit labeling. Specifically, it does so by first learning who to pay attention to (Attention Network), and then learning how to respond to that customer (Interaction Network). To train the Attention Network, we used labels from a Causal Inference Network that learned which customer most likely caused a shopkeeper action. An offline evaluation showed that our proposed system predicted significantly more socially appropriate actions than a baseline system that did not use the Attention Network. A second offline evaluation showed that our procedure for training the Attention Network (using the Causal Inference Network) resulted in significantly more accurate attention predictions than a baseline method that did not use the Causal Inference Network. In the future, we envision that techniques such as this will be crucial in order to teach service robots how to interact with humans in smooth and natural ways.

\section{ACKNOWLEDGMENTS}

We would like to thank the assistants at ATR and Kyoto U. This work was funded by the Japan-United States Educational Commission (Fulbright Japan) and JST CREST Grant Num. JPMJCR17A2, Japan. 


\section{REFERENCES}

[1] Henny Admoni and Brian Scassellati. 2014. Data-driven model of nonverbal behavior for socially assistive human-robot interactions. In Proceedings of the 16th international conference on multimodal interaction. ACM, 196-199.

[2] Dan Bohus and Eric Horvitz. 2009. Learning to Predict Engagement with a Spoken Dialog System in Open-world Settings. In Proceedings of the SIGDIAL 2009 Conference: The 10th Annual Meeting of the Special Interest Group on Discourse and Dialogue (SIGDIAL '09). Association for Computational Linguistics, Stroudsburg, PA, USA, 244-252. http://dl.acm.org/citation.cfm?id=1708376.1708411

[3] Dan Bohus and Eric Horvitz. 2010. Computational Models for Multiparty Turn Taking. Technical Report. Microsoft Research Technical Report MSR-TR 2010115.

[4] Dan Bohus and Eric Horvitz. 2010. Facilitating multiparty dialog with gaze, gesture, and speech. In International Conference on Multimodal Interfaces and the Workshop on Machine Learning for Multimodal Interaction. ACM, 5.

[5] Sonia Chernova, Nick DePalma, Elisabeth Morant, and Cynthia Breazeal. 2011 Crowdsourcing human-robot interaction: Application from virtual to physical worlds. In 2011 RO-MAN. IEEE, 21-26.

[6] Scott Deerwester, Susan T Dumais, George W Furnas, Thomas K Landauer, and Richard Harshman. 1990. Indexing by latent semantic analysis. Fournal of the American society for information science 41, 6 (1990), 391-407.

[7] Malcolm Doering, Dylan F Glas, and Hiroshi Ishiguro. 2019. Modeling Interaction Structure for Robot Imitation Learning of Human Social Behavior. IEEE Transactions on Human-Machine Systems 49, 3 (2019), 219-231.

[8] Malcolm Doering, Phoebe Liu, Dylan F. Glas, Takayuki Kanda, Dana Kulić, and Hiroshi Ishiguro. 2019. Curiosity Did Not Kill the Robot: A Curiosity-based Learning System for a Shopkeeper Robot. ACM Trans. Hum.-Robot Interact. 8, 3 , Article 15 (July 2019), 24 pages. https://doi.org/10.1145/3326462

[9] Dylan F. Glas, Takahiro Miyashita, Hiroshi Ishiguro, and Norihiro Hagita. 2009 Laser-Based Tracking of Human Position and Orientation Using Parametric Shape Modeling. Advanced Robotics 23, 4 (2009), 405-428. https://doi.org/10. 1163/156855309X408754 arXiv:https://doi.org/10.1163/156855309X408754

[10] Randy Gomez, Tatsuya Kawahara, Keisuke Nakamura, and Kazuhiro Nakadai. 2012. Multi-party human-robot interaction with distant-talking speech recognition. In Proceedings of the seventh annual ACM/IEEE international conference on Human-Robot Interaction. ACM, 439-446.

[11] Takuya Iwamoto, Kohtaro Nishi, and Taishi Unokuchi. 2019. Simultaneous Dialog Robot System. In International Conference on Human-Computer Interaction. Springer, 107-111.

[12] Eric Jang, Shixiang Gu, and Ben Poole. 2016. Categorical reparameterization with gumbel-softmax. arXiv preprint arXiv:1611.01144 (2016).

[13] G. F. Jenks. 1967. The Data Model Concept in Statistical Mapping. International Yearbook of Cartography 7 (1967), 186-190.

[14] Martin Johansson and Gabriel Skantze. 2015. Opportunities and obligations to take turns in collaborative multi-party human-robot interaction. In Proceedings of the 16th Annual Meeting of the Special Interest Group on Discourse and Dialogue. 305-314.

[15] Simon Keizer, Mary Ellen Foster, Zhuoran Wang, and Oliver Lemon. 2014. Machine Learning for Social Multiparty Human-Robot Interaction. ACM transactions on interactive intelligent systems (TIIS) 4, 3 (2014), 14

[16] Diederik P Kingma and Jimmy Ba. 2014. Adam: A method for stochastic optimization. arXiv preprint arXiv:1412.6980 (2014).
[17] Andrei P Kirilenko and Svetlana Stepchenkova. 2016. Inter-coder agreement in one-to-many classification: fuzzy kappa. PloS one 11, 3 (2016), e0149787.

[18] Yutaka Kondo, Kentaro Takemura, Jun Takamatsu, and Tsukasa Ogasawara. 2013. A gesture-centric android system for multi-party human-robot interaction. fournal of Human-Robot Interaction 2, 1 (2013), 133-151.

[19] Peter Langfelder, Bin Zhang, and Steve Horvath. 2007. Defining clusters from a hierarchical cluster tree: the Dynamic Tree Cut package for R. Bioinformatics 24, 5 (2007), 719-720.

[20] Phoebe Liu, Dylan F Glas, Takayuki Kanda, and Hiroshi Ishiguro. 2016. Datadriven HRI: Learning social behaviors by example from human-human interaction. IEEE Transactions on Robotics 32, 4 (2016), 988-1008.

[21] Phoebe Liu, Dylan F Glas, Takayuki Kanda, and Hiroshi Ishiguro. 2018. Learning proactive behavior for interactive social robots. Autonomous Robots (2018), 1-19.

[22] James MacQueen et al. 1967. Some methods for classification and analysis of multivariate observations. In Proceedings of the fifth Berkeley symposium on mathematical statistics and probability, Vol. 1. Oakland, CA, USA, 281-297.

[23] Yoichi Morales, Takayuki Kanda, and Norihiro Hagita. 2014. Walking together: Side-by-side walking model for an interacting robot. fournal of Human-Robot Interaction 3, 2 (2014), 50-73.

[24] Bilge Mutlu, Jodi Forlizzi, and Jessica Hodgins. 2006. A storytelling robot: Modeling and evaluation of human-like gaze behavior. In 2006 th IEEE-RAS International Conference on Humanoid Robots. Citeseer, 518-523.

[25] Jeff Orkin and Deb Roy. 2009. Automatic learning and generation of social behavior from collective human gameplay. In Proceedings of The 8th International Conference on Autonomous Agents and Multiagent Systems-Volume 1. International Foundation for Autonomous Agents and Multiagent Systems, 385-392.

[26] Wenxing Quan, Hitoshi Niwa, Naoto Ishikawa, Yoshinori Kobayashi, and Yoshinori Kuno. 2011. Assisted-care robot based on sociological interaction analysis. Computers in Human Behavior 27, 5 (2011), 1527-1534.

[27] Andrea L Thomaz and Crystal Chao. 2011. Turn-taking based on information flow for fluent human-robot interaction. AI Magazine 32, 4 (2011), 53-63.

[28] Rudolph Triebel, Kai Arras, Rachid Alami, Lucas Beyer, Stefan Breuers, Raja Chatila, Mohamed Chetouani, Daniel Cremers, Vanessa Evers, Michelangelo Fiore, et al. 2016. Spencer: A socially aware service robot for passenger guidance and help in busy airports. In Field and service robotics. Springer, 607-622.

[29] Marynel Vázquez, Aaron Steinfeld, and Scott E Hudson. 2015. Parallel detection of conversational groups of free-standing people and tracking of their lower-body orientation. In 2015 IEEE/RS7 International Conference on Intelligent Robots and Systems (IROS). IEEE, 3010-3017.

[30] Marynel Vázquez, Aaron Steinfeld, and Scott E Hudson. 2016. Maintaining awareness of the focus of attention of a conversation: A robot-centric reinforcement learning approach. In 2016 25th IEEE International Symposium on Robot and Human Interactive Communication (RO-MAN). IEEE, 36-43.

[31] Fridolin Wild, Christina Stahl, Gerald Stermsek, and Gustaf Neumann. 2005. Parameters driving effectiveness of automated essay scoring with LSA. (2005).

[32] Chen Yu, Thomas G Smith, Shohei Hidaka, Matthias Scheutz, and Linda B Smith. 2010. A data-driven paradigm to understand multimodal communication in human-human and human-robot interaction. In International Symposium on Intelligent Data Analysis. Springer, 232-244.

[33] Manzil Zaheer, Satwik Kottur, Siamak Ravanbakhsh, Barnabas Poczos, Ruslan R Salakhutdinov, and Alexander J Smola. 2017. Deep sets. In Advances in neural information processing systems. 3391-3401. 